Article

\title{
TBD- or PS-TBD-Catalyzed One-Pot Synthesis of Cyanohydrin Carbonates and Cyanohydrin Acetates from Carbonyl Compounds
}

\author{
Satoru Matsukawa *, Junya Kimura and Miki Yoshioka \\ Department of Science Education, Faculty of Education, Ibaraki University, Ibaraki 310-8512, Japan; \\ varsi10403@gmail.com (J.K.); 13p1419r@vc.ibaraki.ac.jp (M.Y.) \\ * Correspondence: satoru.matsukawa.1@vc.ibaraki.ac.jp; Tel.: +81-29-228-8234; Fax: +81-29-228-8329 \\ Academic Editor: Raquel P. Herrera \\ Received: 30 June 2016; Accepted: 3 August 2016; Published: 10 August 2016
}

\begin{abstract}
Cyanation reactions of carbonyl compounds with methyl cyanoformate or acetyl cyanide catalyzed by $5 \mathrm{~mol} \%$ of 1,5,7-triazabicyclo[4,4,0]dec-5-ene (TBD) were examined. Using methyl cyanoformate, the corresponding cyanohydrin carbonates were readily obtained in high yield for aromatic and aliphatic aldehydes and ketones. Similar results were obtained when acetyl cyanide was used as the cyanide source. The polymer-supported catalyst, PS-TBD, also acted as a good catalyst for this reaction. PS-TBD was easily recovered and reused with minimal activity loss.
\end{abstract}

Keywords: organocatalyst; basecatalyst; guanidine; cyanation; cyanoester

\section{Introduction}

The addition of cyanide to carbonyl compounds is among the most fundamental carbon-carbon bond formation reactions in modern organic chemistry [1-6]. The resultant cyanohydrins are easily converted into important synthetic intermediates including $\alpha$-hydroxyl-carbonyl compounds and $\beta$-amino alcohols. Multiple cyanation methods have been examined, and trimethylsilyl cyanide has most commonly been used as the source of the cyanide anion $[7,8]$. While hundreds of examples have been reported to this day, the inherent instability of trimethylsilyl cyanohydrins sometimes limits the applications of this reaction. Therefore, alternative synthetic procedures for the preparation of stable O-protected cyanohydrins instead of trimethylsilyl cyanohydrin have been developed. Cyanohydrin carbonates and cyanohydrin acylates are configurationally stable and less prone to hydrolysis than cyanohydrin trimethylsilyl ethers. Therefore, one-pot synthesis of cyanohydrin carbonates and acylated cyanation using cyanoformates and cyanoacylates have recently been developed. Several catalytic reactions have been recently studied. To date, reactions using tributyltin cyanide [9], $\mathrm{K}_{2} \mathrm{CO}_{3}$ [10], tertiary amines [11,12], DABCO (1,4-diazabicyclo[2.2.2]octane) [13,14], dimethyl sulfoxide [15], TTMPP (tris(2,4,6-trimethoxyphenyl)phsphine) [16], DMAP ( $N, N$-dimethyl-4-aminopyridine) [17], and $N$-heterocyclic carbenes [18] as catalysts or activators have been reported. Asymmetric catalytic reactions have also been reported using cinchona alkaloids [19-25], Y complexes [26-28], Al complexes [29,30], Ti complexes [31-40], and V complexes [41]. To further extend the scope of this versatile synthetic reaction, we have now examined the use of 1,5,7-triazabicyclo[4.4.0]dec-5-ene (TBD) as a Lewis base catalyst.

It is well-known that TBD is a strong Lewis base owing to its distorted bicyclic guanidine structure [42,43]. Employing this property, multiple unique reactions have been reported using TBD as an organobase catalyst including Henry reactions [44], Wittig and Horner-Wadsworth-Emmons reactions [45], Michael reactions [46,47], intramolecular aldol reactions [48,49], ring-opening polymerization [50,51], aminolysis of esters [52,53], conjugate addition 
to activated alkenes [54], synthesis of pyrazolines [55], and others [56,57]. Although TBD usually behaves as a base, it also contains an acidic N-H group; therefore, TBD may act as a bifunctional acid-base catalyst [58-60]. Recently, we have reported the trifluoromethylation of aldehydes [61] and the ring-opening reactions of aziridines [62,63] catalyzed using TBD as an organocatalyst. Here, we report that TBD acts as an effective organobase catalyst for the cyanation of carbonyl compounds using methyl cyanoformate or acetyl cyanide as cyanide source.

\section{Results and Discussion}

Initially, the cyanomethoxycarbonylation reaction of $p$-anisaldehyde using methyl cyanoformate was examined in the presence of $5 \mathrm{~mol} \%$ of TBD in THF at room temperature. The desired product was obtained in $90 \%$ yield after $2 \mathrm{~h}$ (Table 1, entry 1). The product was obtained in a lower yield when DMF (N,N-dimethylformamide), toluene or $\mathrm{ClCH}_{2} \mathrm{CH}_{2} \mathrm{Cl}$ was used as the solvent. An approximately comparable yield was observed when $\mathrm{CH}_{3} \mathrm{CN}$ was used (Table 1, entry 2). The yield was decreased when $1 \mathrm{~mol} \%$ of TBD was used (Table 1, entry 6). To clarify the scope of this reaction, the cyanation of several aldehydes was examined in the presence of $5 \mathrm{~mol} \%$ of TBD. In the case of aromatic aldehydes, the yield was influenced by the substituents on the aromatic ring. With aromatic aldehydes bearing an electron-withdrawing group, it was necessary to change the order of reagent addition to obtain the desired product in good yield. Using other aromatic aldehydes, the products were obtained in good to high yields. trans-Cinnamaldehyde was subjected to our reaction conditions, and the 1,2-addition product was obtained in $85 \%$ yield. Although slightly longer reaction times were required, aliphatic aldehydes also produced similar high yields.

Table 1. TBD (1,5,7-triazabicyclo[4,4,0]dec-5-ene) catalyzed cyanation of aldehydes with methyl cyanoformate.

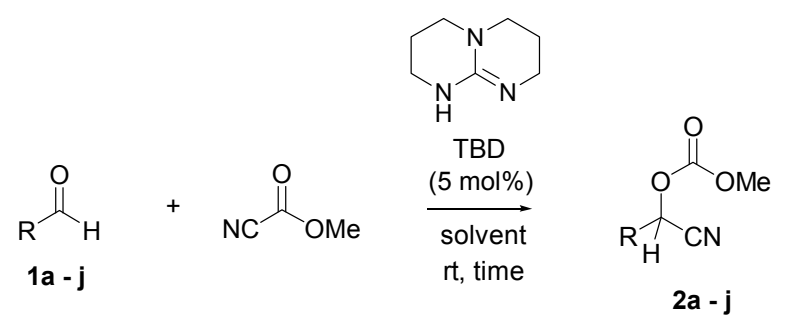

\begin{tabular}{|c|c|c|c|c|c|}
\hline Entry & Aldehyde(1) & Product & Solvent & Time & Yield (\%) ${ }^{a}$ \\
\hline 1 & & $2 a$ & $\mathrm{CH}_{3} \mathrm{CN}$ & $2 \mathrm{~h}$ & 90 \\
\hline 2 & & & THF & $2 \mathrm{~h}$ & 81 \\
\hline 3 & & & $\mathrm{ClCH}_{2} \mathrm{CH}_{2} \mathrm{Cl}$ & $3 \mathrm{~h}$ & 65 \\
\hline 4 & & & DMF & $3 \mathrm{~h}$ & 60 \\
\hline 5 & & & toluene & $12 \mathrm{~h}$ & 35 \\
\hline $6^{b}$ & & & $\mathrm{CH}_{3} \mathrm{CN}$ & $12 \mathrm{~h}$ & 68 \\
\hline 7 & & $2 b$ & $\mathrm{CH}_{3} \mathrm{CN}$ & $12 \mathrm{~h}$ & 89 \\
\hline 8 & & $2 c$ & & $4 \mathrm{~h}$ & 92 \\
\hline
\end{tabular}


Table 1. Cont.

\begin{tabular}{|c|c|c|c|c|c|}
\hline Entry & Aldehyde(1) & Product & Solvent & Time & Yield (\%) ${ }^{a}$ \\
\hline $9^{c}$ & $\mathrm{CHC}$ & $2 d$ & & $0.5 \mathrm{~h}$ & 70 \\
\hline 10 & $\mathrm{CH}$ & $2 e$ & & $4 \mathrm{~h}$ & 96 \\
\hline 11 & $\mathrm{CHO}$ & $2 f$ & & $4 \mathrm{~h}$ & 90 \\
\hline 12 & & $2 g$ & & $2 \mathrm{~h}$ & 85 \\
\hline 13 & & $2 h$ & & $4 \mathrm{~h}$ & 75 \\
\hline 14 & $\mathrm{CHO}$ & $2 \mathbf{i}$ & & $4 \mathrm{~h}$ & 96 \\
\hline 15 & $\mathrm{n}-\mathrm{C}_{8} \mathrm{H}_{17} \mathrm{CHO}$ & $2 j$ & & $4 \mathrm{~h}$ & 89 \\
\hline 16 & & 2k & & $4 \mathrm{~h}$ & 88 \\
\hline
\end{tabular}

Ketones were also examined using the $10 \mathrm{~mol} \%$ of TBD (Table 2). In the case of aliphatic ketones, good results were obtained for both acyclic and cyclic ketones. The reaction proceeded more smoothly than the reported example [17]. Only 1,2-addition products were observed for unsaturated ketones. On the other hand, for aromatic ketones, only a small amount of the desired product was obtained at room temperature even after $24 \mathrm{~h}$. When the reactions were performed at elevated temperature $\left(50^{\circ} \mathrm{C}\right)$, a variety of aromatic ketones were converted to their corresponding cyanohydrin carbonates in moderate to good yields. In this reaction, novel cyanohydrin carbonates (4c, $4 \mathbf{g})$ could be obtained. In addition, $4 \mathbf{d}$ and $4 \mathbf{e}$ were obtained for the first time as products of this one-pot method. Unfortunately, in the reaction of 4-methoxyacetophenone, no product was found under these conditions.

Table 2. TBD catalyzed cyanation of ketones with methyl cyanoformate.

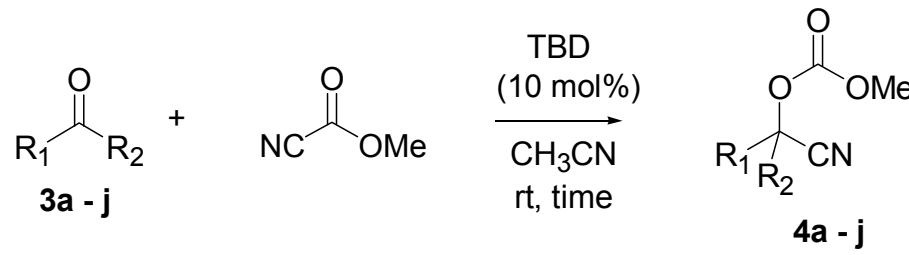

\begin{tabular}{crccc}
\hline Entry & Ketone(3) & Product & Time & Yield (\%) $^{\mathbf{a}}$ \\
\hline 1 & 0 & & & \\
& & $4 \mathbf{a}$ & $4 \mathrm{~h}$ & 85 \\
\hline 2 & $\mathrm{O}$ & $4 \mathbf{b}$ & $1 \mathrm{~h}$ & 90 \\
\hline
\end{tabular}


Table 2. Cont

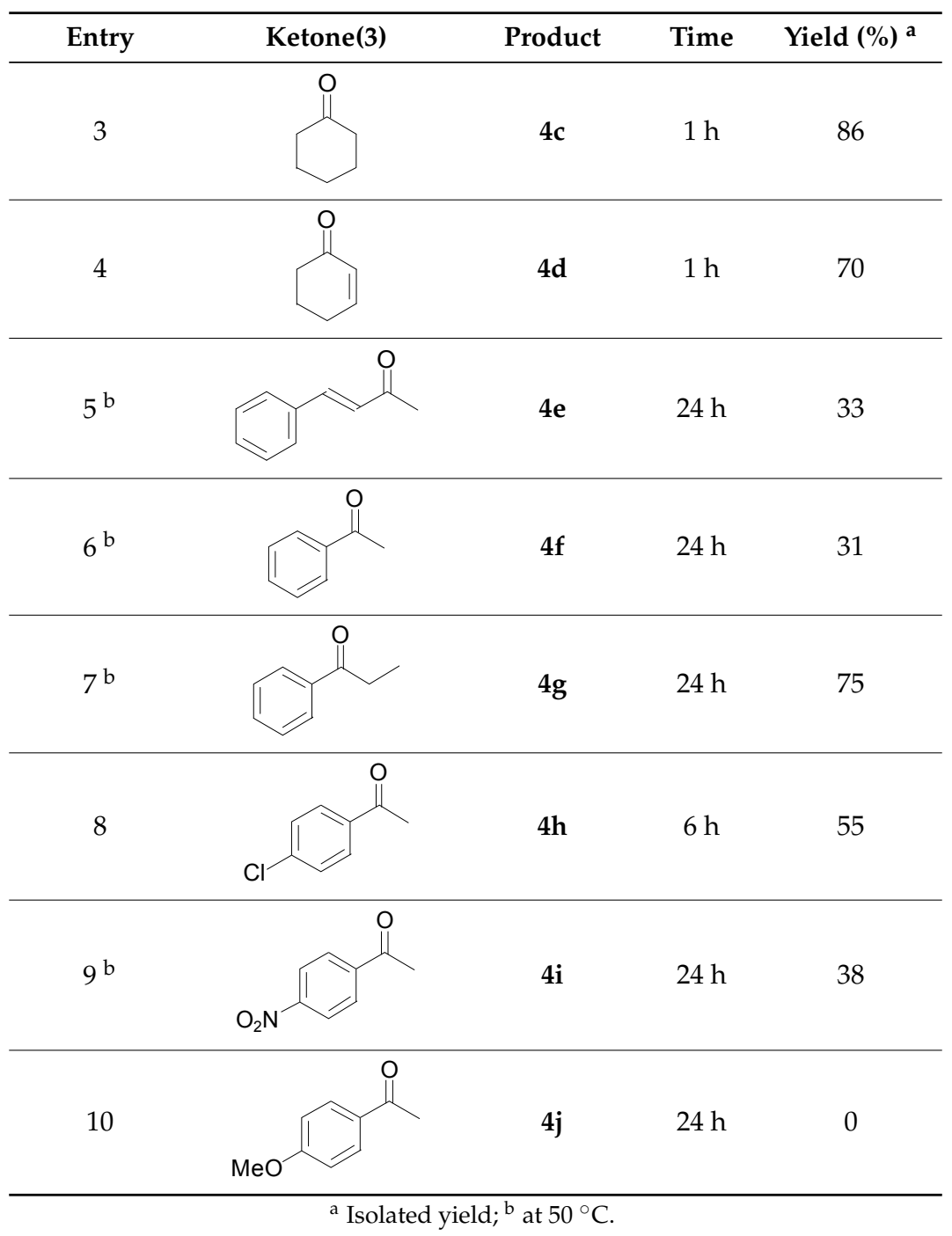

Acetyl cyanide was also examined in the TBD-catalyzed reaction (Table 3). The acylated cyanohydrin products were readily obtained in high yields in a similar manner for aromatic aldehydes. Although the bond between the acetyl and cyanide groups is stronger in acetyl cyanide than in methyl cyanoformate, the reactivity did not decrease. In the case of 4-nitrobenzaldehyde, the starting material was rapidly consumed; however, the product yield was not good. In this case, it is likely that attack at the $\mathrm{NO}_{2}$ group occurred. Longer reaction times were needed in the reaction of aromatic aldehydes bearing electron-donating groups on the aromatic ring. This trend has been similarly reported in a recent related report [18]. The aliphatic, heterocyclic, and $\alpha, \beta$-unsaturated aldehydes also reacted to give the corresponding products in similarly high yields.

Furthermore, we applied a polymer-supported TBD, PS-TBD [64-67] to this reaction (Table 4). Polymer-supported catalysts have attracted significant attention in recent decades because of their inherent advantages in synthetic chemistry, e.g., the simplification of reaction procedures including the easy recovery of the catalyst using filtration, application to automated systems, and recycling of the catalyst [68-73]. Thus, we examined the cyanomethoxycarbonylation reaction of $p$-anisaldehydes in the presence of $10 \mathrm{~mol} \%$ of PS-TBD [63,74-79]. The reaction proceeded smoothly and the desired product was obtained in $95 \%$ yield after $3 \mathrm{~h}$ at room temperature. A variety of aldehydes reacted well with methyl cyanoformate to give cyanohydrin carbonates. Aromatic aldehydes with either electron-donating or electron-withdrawing groups afforded the corresponding products in high yields. 
Aliphatic aldehydes also worked well. In the reaction with aldehyde, the catalytic reactivity of PS-TBD seems to be slightly lower than TBD. Ketones were also examined; however, the reaction was very slow. Then, the reaction was performed at elevated temperature. The desired product was obtained at moderate to yield in $4 \mathrm{~h}$ at $50{ }^{\circ} \mathrm{C}$. In addition, the recovery and reuse of PS-TBD for the reaction of $p$-anisaldehyde with methyl cyanoformate was also examined. After the reaction was completed, ethyl acetate was added to the reaction mixture and the catalyst was recovered by filtration. The recovered catalyst was washed, dried and then reused. The catalyst was reused, maintaining its catalytic activity after three times.

Table 3. TBD catalyzed cyanation of aldehydes with acetyl cyanide.

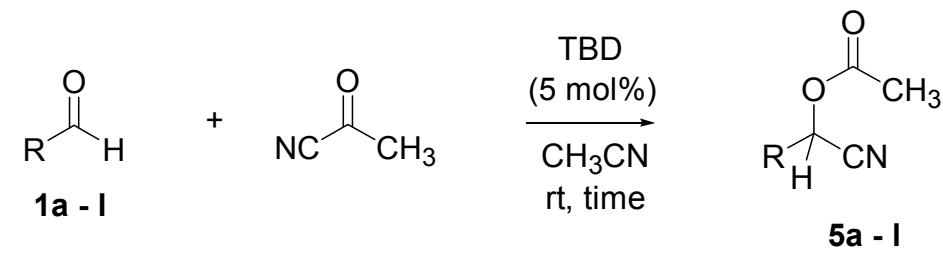

\begin{tabular}{|c|c|c|c|c|}
\hline Entry & Aldehyde(1) & Product & Time & Yield (\%) ${ }^{a}$ \\
\hline 1 & $\mathrm{HOO}$ & $5 a$ & $3 \mathrm{~h}$ & 80 \\
\hline 2 & $\mathrm{CHO}$ & $5 b$ & $6 \mathrm{~h}$ & 91 \\
\hline 3 & $\mathrm{HC}$ & $5 c$ & $24 \mathrm{~h}$ & 94 \\
\hline 4 & $\mathrm{CH}$ & $5 d$ & $48 \mathrm{~h}$ & 67 \\
\hline 5 & & $5 e$ & $0.5 \mathrm{~h}$ & 40 \\
\hline 6 & $\mathrm{CHC}$ & $5 f$ & $3 \mathrm{~h}$ & 90 \\
\hline 7 & $\mathrm{CHO}$ & $5 \mathrm{~g}$ & $3 \mathrm{~h}$ & 90 \\
\hline 8 & & $5 \mathrm{~h}$ & $3 \mathrm{~h}$ & 92 \\
\hline 9 & & $5 i$ & $6 \mathrm{~h}$ & 70 \\
\hline 10 & $\mathrm{CHO}$ & $5 j$ & $6 \mathrm{~h}$ & 88 \\
\hline 11 & $\mathrm{n}-\mathrm{C}_{8} \mathrm{H}_{17} \mathrm{CHO}$ & $5 k$ & $6 \mathrm{~h}$ & 85 \\
\hline 12 & & 51 & $6 \mathrm{~h}$ & 80 \\
\hline
\end{tabular}


Table 4. PS (polymer-supported)-TBD catalyzed cyanation of carbonyl compounds with methyl cyanoformate.

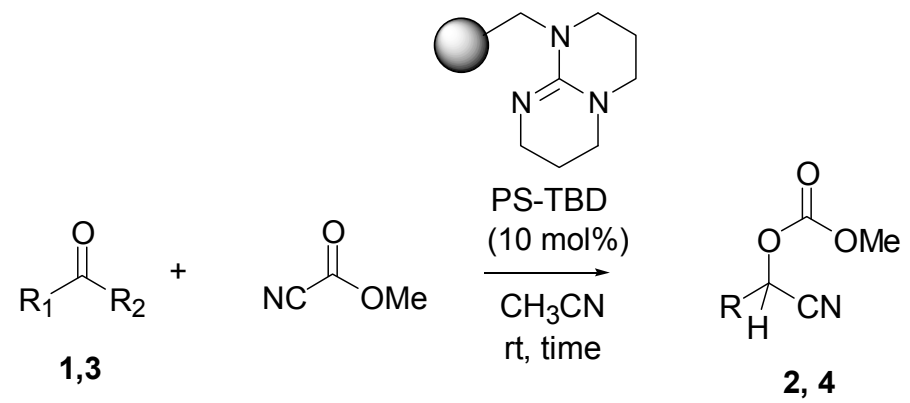

\begin{tabular}{|c|c|c|c|c|}
\hline Entry & Carbonyl Compounds & Product & Time & Yield (\%) ${ }^{a}$ \\
\hline & $\mathrm{HO}$ & & & \\
\hline 1 & & $2 a$ & $2 \mathrm{~h}$ & 90 \\
\hline $2^{b}$ & & $2 a$ & $2 \mathrm{~h}$ & 88 \\
\hline $3^{c}$ & & $2 a$ & $2 \mathrm{~h}$ & 92 \\
\hline $4^{\mathrm{d}}$ & & $2 a$ & $2 \mathrm{~h}$ & 88 \\
\hline 5 & 8 & $2 b$ & $12 \mathrm{~h}$ & 73 \\
\hline & $\mathrm{HO}$ & & & \\
\hline 6 & & $2 c$ & $16 \mathrm{~h}$ & 86 \\
\hline & & & & \\
\hline 7 & & $2 d$ & $4 \mathrm{~h}$ & 94 \\
\hline & $\mathrm{HO}$ & & & \\
\hline 8 & & $2 e$ & $4 \mathrm{~h}$ & 98 \\
\hline 9 & & $2 h$ & $4 \mathrm{~h}$ & 90 \\
\hline 10 & & 2k & $2 \mathrm{~h}$ & 78 \\
\hline 11 & & $2 \mathbf{i}$ & $2 \mathrm{~h}$ & 82 \\
\hline 12 & & $4 a$ & $24 \mathrm{~h}$ & 24 \\
\hline $13^{\mathrm{e}}$ & & $4 a$ & $12 \mathrm{~h}$ & 75 \\
\hline $14^{\mathrm{e}}$ & & $4 b$ & $24 \mathrm{~h}$ & 70 \\
\hline $15^{\mathrm{e}}$ & & $4 c$ & $18 \mathrm{~h}$ & 76 \\
\hline
\end{tabular}

${ }^{\mathrm{a}}$ Isolated yield; ${ }^{\mathrm{b}}$ 2nd run; ${ }^{\mathrm{c}} 3 \mathrm{rd}^{\mathrm{run}} ;^{\mathrm{d}} 4$ th run; ${ }^{\mathrm{e}}$ at $50^{\circ} \mathrm{C}$. 
A possible mechanism for the TBD-catalyzed cyanation is illustrated in Scheme 1. First, the nitrogen atom of TBD activates the cyanoformates or cyanoacylates to form $\left[\mathrm{N}^{+} \mathrm{C}(\mathrm{O}) \mathrm{R}\right] \mathrm{CN}{ }^{-}$ intermediate A. Next, the $\mathrm{CN}^{-}$anion immediately reacts with the carbonyl compounds to give the alkoxide anion of the cyanohydrin B. Finally, the alkoxide anion is carbonylated or acylated to give the $\mathrm{O}$-protected cyanohydrin with the regeneration of TBD. In this reaction, acidic $\mathrm{N}-\mathrm{H}$ group of TBD may activate the carbonyl group $(\mathbf{C})$.

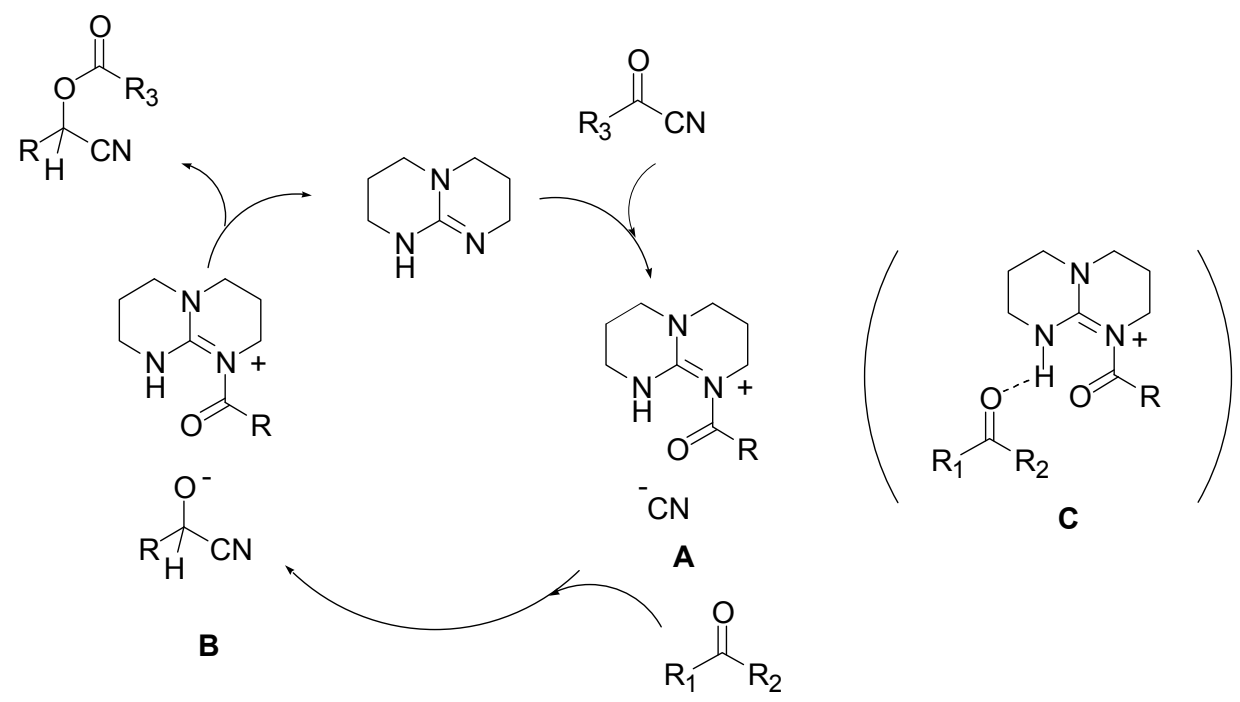

Scheme 1. Proposed mechanism. $\left[\mathrm{N}^{+} \mathrm{C}(\mathrm{O}) \mathrm{R}\right] \mathrm{CN}^{-}$intermediate $\mathbf{A}$; alkoxide anion of the cyanohydrin $\mathbf{B}$ and alternative transition state $\mathbf{C}$.

\section{Experimental Section}

\subsection{General}

IR spectra were recorded on a JUSCO FT/IR-430 spectrometer (JASCO Corporation, Tokyo, Japan). ${ }^{1} \mathrm{H}-\mathrm{NMR}$ spectra were determined for solutions in $\mathrm{CDCl}_{3}$ with $\mathrm{Me}_{4} \mathrm{Si}$ as internal standard $\left(\mathrm{CDCl}_{3}: 7.24 \mathrm{ppm}, \mathrm{Me}_{4} \mathrm{Si}: 0.00 \mathrm{ppm}\right)$ on a Bruker Avance III instrument (Bruker Corporation, Billerica, MA, USA). ${ }^{13} \mathrm{C}-\mathrm{NMR}$ spectra were determined for solutions in $\mathrm{CDCl}_{3}$ with $\mathrm{Me}_{4} \mathrm{Si}$ as internal standard $\left(\mathrm{CDCl}_{3}\right.$ : $\left.77.00 \mathrm{ppm}, \mathrm{Me}_{4} \mathrm{Si}: 0.00 \mathrm{ppm}\right)$ on a Bruker Avance III instrument (Bruker Corporation). HRMS data were measured on a JEOL JMS-700 mass spectrometer (JEOL Ltd., Tokyo, Japan).

All reactions were performed under an argon atmosphere using oven-dried glassware. Flash column chromatography was performed using silica gel Wakogel C-200 (Wako Chemical, Osaka, Japan). Dehydrate DMF, THF, toluene and $\mathrm{CH}_{3} \mathrm{CN}$ were purchased from Kanto Kagaku (Tokyo, Japan) as the "anhydrous" and stored over 4A molecular sieves under Ar. Other commercially available reagent was used as received without further purification.

\subsection{Method}

3.2.1. General Procedure for TBD-Catalyzed Cyanation Reactions of Carbonyl Compounds with Methyl Cyanoformate

TBD was dried in vacuo before use. To a solution of TBD $(0.05 \mathrm{mmol})$ in $\mathrm{CH}_{3} \mathrm{CN}(1 \mathrm{~mL})$ was added carbonyl compound $(1.0 \mathrm{mmol})$ and methyl cyanoformate $(1.25 \mathrm{mmol})$ at room temperature. After the reaction was complete (as determined by TLC), the reaction mixture was quenched with water. The resultant mixture was extracted with EtOAc $(3 \times 10 \mathrm{~mL})$. The combined organic layers were washed with water and brine and dried with $\mathrm{Na}_{2} \mathrm{SO}_{4}$. After the filtration, the residue was 
concentrated in vacuo. The crude product was purified by column chromatography on silica gel (EtOAc:hexane $=1: 5 \rightarrow 1: 3$ ) to give the corresponding product.

3.2.2. General Procedure for TBD-Catalyzed Cyanation Reactions of Aldehydes with Acetyl Cyanide

TBD was dried in vacuo before use. To a solution of TBD $(0.10 \mathrm{mmol})$ in $\mathrm{CH}_{3} \mathrm{CN}(1 \mathrm{~mL})$ was added aldehyde $(1.0 \mathrm{mmol})$ and acetyl cyanide $(1.5 \mathrm{mmol})$ at room temperature. After the reaction was complete (as determined by TLC), the reaction mixture was quenched with water. The resultant mixture was extracted with EtOAc $(3 \times 10 \mathrm{~mL})$. The combined organic layers were washed with water and brine and dried with $\mathrm{Na}_{2} \mathrm{SO}_{4}$. After the filtration, the residue was concentrated in vacuo. The crude product was purified by column chromatography on silica gel (EtOAc:hexane $=1: 10 \rightarrow 1: 5$ ) to give the corresponding product.

3.2.3. General Procedure for PS-TBD Catalyzed Cyanation Reactions of Carbonyl Compounds with Methyl Cyanoformate

PS-TBD was dried in vacuo before use. To a solution of PS-TBD $(0.10 \mathrm{mmol})$ in $\mathrm{CH}_{3} \mathrm{CN}(1 \mathrm{~mL})$ was added carbonyl compound $(1.0 \mathrm{mmol})$ and methyl cyanoformate $(1.25 \mathrm{mmol})$ at room temperature. After the reaction was complete (as determined by TLC), EtOAc $(10 \mathrm{~mL})$ was added to the mixture and PS-TBD was separated by filtration. The filtrate was washed with water and brine and dried with $\mathrm{Na}_{2} \mathrm{SO}_{4}$. After the filtration, the residue was concentrated in vacuo. The crude product was purified by column chromatography on silica gel (EtOAc:hexane $=1: 5 \rightarrow 1: 3$ ) to give the corresponding product. The recovered catalyst is reusable after washing (acetone and water) and drying in vacuo.

\subsection{General Characterization of the Products}

Cyano(4-methoxyphenyl)methyl methyl carbonate (2a) [11]: IR (neat): 2245, $1761 \mathrm{~cm}^{-1} ;{ }^{1} \mathrm{H}-\mathrm{NMR}(500 \mathrm{MHz}$, $\left.\mathrm{CDCl}_{3}\right): \delta 3.80(\mathrm{~s}, 3 \mathrm{H}), 3.82,(\mathrm{~s}, 3 \mathrm{H}), 6.18(\mathrm{~s}, 1 \mathrm{H}), 6.92(\mathrm{~d}, J=8.8 \mathrm{~Hz}, 2 \mathrm{H}), 7.44(\mathrm{~d}, J=8.8 \mathrm{~Hz}, 2 \mathrm{H})$; ${ }^{13} \mathrm{C}-\mathrm{NMR}\left(125 \mathrm{MHz}, \mathrm{CDCl}_{3}\right): \delta 55.5,55.7,66.4,114.6,115.9,123.2,129.8,154.1,161.4$. The corresponding spectra are shown in Supplementary Materials.

Cyano(phenyl)methyl methyl carbonate (2b) [11]: IR (neat): $2249,1760 \mathrm{~cm}^{-1},{ }^{1} \mathrm{H}-\mathrm{NMR}\left(500 \mathrm{MHz}, \mathrm{CDCl}_{3}\right)$ : $\delta 3.77(\mathrm{~s}, 3 \mathrm{H}), 6.18(\mathrm{~s}, 1 \mathrm{H}), 7.35-7.46(\mathrm{~m}, 5 \mathrm{H}) ;{ }^{13} \mathrm{C}-\mathrm{NMR}\left(125 \mathrm{MHz}, \mathrm{CDCl}_{3}\right): \delta 55.9,66.6,115.7,127.9$, $129.3,130.7,131.1,154.1$.

Cyano(4-chlorophenyl)methyl methyl carbonate (2c) [11]: IR (neat): 2250, $1758 \mathrm{~cm}^{-1} ; 2347,1762 \mathrm{~cm}^{-1}$; ${ }^{1} \mathrm{H}-\mathrm{NMR}\left(500 \mathrm{MHz}, \mathrm{CDCl}_{3}\right): \delta 3.84(\mathrm{~s}, 3 \mathrm{H}), 6.21(\mathrm{~s}, 1 \mathrm{H}), 7.40(\mathrm{~d}, J=8.6 \mathrm{~Hz}, 2 \mathrm{H}), 7.46(\mathrm{~d}, J=8.6 \mathrm{~Hz}, 2 \mathrm{H})$; ${ }^{13} \mathrm{C}-\mathrm{NMR}\left(125 \mathrm{MHz}, \mathrm{CDCl}_{3}\right): \delta 56.0,65.8,115.3,129.2,129.3,129.6,136.9,153.9$.

Cyano(4-nitrophenyl)methyl methyl carbonate (2d) [16]: IR (neat): 2251, $1760 \mathrm{~cm}^{-1} ;{ }^{1} \mathrm{H}-\mathrm{NMR}(500 \mathrm{MHz}$, $\left.\mathrm{CDCl}_{3}\right): \delta 2.20(\mathrm{~s}, 3 \mathrm{H}), 6.49(\mathrm{~s}, 1 \mathrm{H}), 7.71(\mathrm{~d}, J=8.7 \mathrm{~Hz}, 2 \mathrm{H}), 8.30(\mathrm{~d}, J=8.7 \mathrm{~Hz}, 2 \mathrm{H}) ;{ }^{13} \mathrm{C}-\mathrm{NMR}(125 \mathrm{MHz}$, $\left.\mathrm{CDCl}_{3}\right): 20.3,61.6,115.1,124.4,128.7,138.1,149.0,168.6$.

Cyano(naphthalen-1-yl)methyl methyl carbonate (2e) [15]: IR (neat): 2247, $1768 \mathrm{~cm}^{-1} ;{ }^{1} \mathrm{H}-\mathrm{NMR}(500 \mathrm{MHz}$, $\left.\mathrm{CDCl}_{3}\right): \delta 3.77(\mathrm{~s}, 3 \mathrm{H}), 6.18(\mathrm{~s}, 1 \mathrm{H}), 7.51-7.58(\mathrm{~m}, 3 \mathrm{H}), 7.80-7.96(\mathrm{~m}, 4 \mathrm{H}) ;{ }^{13} \mathrm{C}-\mathrm{NMR}\left(125 \mathrm{MHz}, \mathrm{CDCl}_{3}\right)$ : $\delta 56.0,65.3,115.8,122.6,125.0,126.4,127.7,129.1,129.2,130.0,131.8,133.9,154.2$.

Cyano(naphthalen-2-yl)methyl methyl carbonate (2f) [15]: IR (neat): 2254, $1757 \mathrm{~cm}^{-1} ;{ }^{1} \mathrm{H}-\mathrm{NMR}(500 \mathrm{MHz}$, $\left.\mathrm{CDCl}_{3}\right): \delta 3.85(\mathrm{~s}, 3 \mathrm{H}), 6.42(\mathrm{~s}, 1 \mathrm{H}), 7.51-7.56(\mathrm{~m}, 3 \mathrm{H}), 7.84-7.96(\mathrm{~m}, 4 \mathrm{H}) ;{ }^{13} \mathrm{C}-\mathrm{NMR}\left(125 \mathrm{MHz}, \mathrm{CDCl}_{3}\right):$ $\delta 55.9,66.8,115.8,124.1,127.1,127.7,127.9,128.1,128.4,128.5,129.5,132.8,134.0,154.2$.

Cyano-3-phenylpropen-2-yl methyl carbonate (2g) [15]: IR (neat): 2260, $1759 \mathrm{~cm}^{-1}$; ${ }^{1} \mathrm{H}-\mathrm{NMR}(500 \mathrm{MHz}$, $\left.\mathrm{CDCl}_{3}\right): \delta 3.86(\mathrm{~s}, 3 \mathrm{H}), 5.88(\mathrm{dd}, J=1.1,6.8 \mathrm{~Hz}, 1 \mathrm{H}), 6.20(\mathrm{dd}, J=6.8,15.8 \mathrm{~Hz}, 1 \mathrm{H}), 6.98(\mathrm{~d}, J=15.8 \mathrm{~Hz}$, 
1H), 7.33-7.38 (m, 3H), 7.40-7.46 (m, 2H); ${ }^{13} \mathrm{C}-\mathrm{NMR}\left(125 \mathrm{MHz}, \mathrm{CDCl}_{3}\right): \delta 55.9,65.2,114.9,117.6,127.2$, $128.2,129.5,134.2,138.4,154.0$.

Cyano-3-phenylpropyl methyl carbonate (2h) [15]: IR (neat): 2240, $1760 \mathrm{~cm}^{-1},{ }^{1} \mathrm{H}-\mathrm{NMR}\left(500 \mathrm{MHz}, \mathrm{CDCl}_{3}\right)$ : $\delta 2.22-2.30(\mathrm{~m}, 2 \mathrm{H}), 2.85(\mathrm{t}, J=7.8 \mathrm{~Hz}, 2 \mathrm{H}), 3.86(\mathrm{~s}, 3 \mathrm{H}), 5.13(\mathrm{t}, J=7.8 \mathrm{~Hz}, 2 \mathrm{H}), 7.18-7.21(\mathrm{~m}, 3 \mathrm{H})$, 7.29-7.33 (m, 2H); ${ }^{13} \mathrm{C}-\mathrm{NMR}\left(125 \mathrm{MHz}, \mathrm{CDCl}_{3}\right): \delta 30.4,33.8,55.8,64.0,116.2,126.7,128.2,128.4,128.7$, 129.0, 138.7, 154.0.

Cyano(cyclohexyl)methyl methyl carbonate (2i) [15]: IR (neat): 2247, $1758 \mathrm{~cm}^{-1} ;{ }^{1} \mathrm{H}-\mathrm{NMR}(500 \mathrm{MHz}$, $\left.\mathrm{CDCl}_{3}\right): \delta 1.06-1.25(\mathrm{~m}, 5 \mathrm{H}), 1.65(\mathrm{~d}, J=12.7 \mathrm{~Hz}, 1 \mathrm{H}), 1.89(\mathrm{~m}, 6 \mathrm{H}), 3.79(\mathrm{~s}, 3 \mathrm{H}), 4.98(\mathrm{~d}, J=6.0 \mathrm{~Hz}$, $1 \mathrm{H}) 3.80(\mathrm{~s}, 3 \mathrm{H}), 6.28(\mathrm{~s}, 1 \mathrm{H}), 6.37(\mathrm{dd}, J=1.9,3.4 \mathrm{~Hz}, 1 \mathrm{H}), 6.66(\mathrm{~d}, J=3.4 \mathrm{~Hz}, 1 \mathrm{H}), 7.45(\mathrm{~m}, 1 \mathrm{H})$; ${ }^{13} \mathrm{C}-\mathrm{NMR}\left(125 \mathrm{MHz}, \mathrm{CDCl}_{3}\right): \delta 25.2,27.7,27.9,40.1,55.7,69.5,115.7,154.3$.

Cyano(1-octyl)methyl methyl carbonate (2j) [16]: IR (neat): 2249, $1760 \mathrm{~cm}^{-1} ;{ }^{1} \mathrm{H}-\mathrm{NMR}\left(500 \mathrm{MHz}, \mathrm{CDCl}_{3}\right.$ ): $\delta 0.81(\mathrm{~m}, 3 \mathrm{H}), 1.19-1.21(\mathrm{~m}, 12 \mathrm{H}), 1.87(\mathrm{dd}, J=6.8,15.8 \mathrm{~Hz}, 2 \mathrm{H}), 3.79(\mathrm{~s}, 3 \mathrm{H}), 5.13(\mathrm{t}, J=6.8 \mathrm{~Hz}, 1 \mathrm{H})$; ${ }^{13} \mathrm{C}-\mathrm{NMR}\left(125 \mathrm{MHz}, \mathrm{CDCl}_{3}\right)$ : $\delta$ 14.0, 22.6, 24.4, 28.8, 29.2, 29.5, 31.7, 32.3, 55.7, 64.9, 116.5, 154.3.

Cyano(furan-2-yl)methyl methyl carbonate (2k) [11]: IR (neat): 2247, $1768 \mathrm{~cm}^{-1} ;{ }^{1} \mathrm{H}-\mathrm{NMR}(500 \mathrm{MHz}$, $\left.\mathrm{CDCl}_{3}\right): \delta 3.80(\mathrm{~s}, 3 \mathrm{H}), 6.28(\mathrm{~s}, 1 \mathrm{H}), 6.37(\mathrm{dd}, J=1.9,3.4 \mathrm{~Hz}, 1 \mathrm{H}), 6.66(\mathrm{~d}, J=3.4 \mathrm{~Hz}, 1 \mathrm{H}), 7.45(\mathrm{~m}, 1 \mathrm{H})$; ${ }^{13} \mathrm{C}-\mathrm{NMR}\left(125 \mathrm{MHz}, \mathrm{CDCl}_{3}\right): \delta 56.0,59.3,111.2,113.2,143.5,145.4,153.9$.

1-Cyano-1-methyl-3-phenylpropyl methyl carbonate (4a) [11]: IR (neat): 2250, $1760 \mathrm{~cm}^{-1}$; ${ }^{1} \mathrm{H}-\mathrm{NMR}\left(500 \mathrm{MHz}, \mathrm{CDCl}_{3}\right): \delta 1.82(\mathrm{~s}, 3 \mathrm{H}), 2.17-2.34(\mathrm{~m}, 2 \mathrm{H}), 2.82-2.90(\mathrm{~m}, 2 \mathrm{H}), 3.83(\mathrm{~s}, 3 \mathrm{H})$, 7.16-7.24 (m, 3H), 7.26-7.31 (m, 2H); ${ }^{13} \mathrm{C}-\mathrm{NMR}\left(125 \mathrm{MHz}, \mathrm{CDCl}_{3}\right): \delta 24.5,30.2,41.6,55.3,74.0,118.2$, $126.5,128.4,128.7,139.7,153.2$.

1-Cyano-1-ethylhexyl methyl carbonate (4b) [16]: IR (neat): 2250, $1759 \mathrm{~cm}^{-1} ;{ }^{1} \mathrm{H}-\mathrm{NMR}\left(500 \mathrm{MHz}, \mathrm{CDCl}_{3}\right)$ : $\delta 0.85(\mathrm{t}, J=6.9 \mathrm{~Hz}, 3 \mathrm{H}), 1.03(\mathrm{t}, J=7.5 \mathrm{~Hz}, 3 \mathrm{H}), 1.24-1.33(\mathrm{~m}, 4 \mathrm{H}), 1.35-1.54(\mathrm{~m}, 2 \mathrm{H}), 1.88-2.10(\mathrm{~m}, 4 \mathrm{H})$, $3.78(\mathrm{~s}, 3 \mathrm{H}) ;{ }^{13} \mathrm{C}-\mathrm{NMR}\left(125 \mathrm{MHz}, \mathrm{CDCl}_{3}\right): \delta$ 7.9, 13.8, 22.3, 23.2, 29.8, 31.3, 36.0, 55.1, 78.5, 117.8, 153.2 .

1-Cyanocyclohexyl methyl carbonate (4c): colorless oil; IR (neat): 2250, $1760 \mathrm{~cm}^{-1} ;{ }^{1} \mathrm{H}-\mathrm{NMR}$ ( $500 \mathrm{MHz}$, $\left.\mathrm{CDCl}_{3}\right): \delta 1.15-1.35(\mathrm{~m}, 1 \mathrm{H}), 1.53-1.64(\mathrm{~m}, 3 \mathrm{H}), 1.70-1.77(\mathrm{~m}, 2 \mathrm{H}), 1.80-1.85(\mathrm{~m}, 2 \mathrm{H}), 2.26-2.28(\mathrm{~m}, 2 \mathrm{H})$, $3.79(\mathrm{~s}, 3 \mathrm{H}) ;{ }^{13} \mathrm{C}-\mathrm{NMR}\left(125 \mathrm{MHz}, \mathrm{CDCl}_{3}\right): \delta 22.0,24.3,35.0,55.1,75.0,118.2,153.0 ;$ HRMS (FAB): $m / z$ : calcd for $\mathrm{C}_{9} \mathrm{H}_{14} \mathrm{NO}_{3}$ : 184.0973; found: $184.0991[\mathrm{M}+\mathrm{H}]^{+}$.

1-cyanocyclohex-2-en-1-yl methyl carbonate (4d) [80]: IR (neat): 2245, $1753 \mathrm{~cm}^{-1} ;{ }^{1} \mathrm{H}-\mathrm{NMR}(500 \mathrm{MHz}$, $\left.\mathrm{CDCl}_{3}\right): \delta 1.68-72(\mathrm{~m}, 2 \mathrm{H}), 1.97-1.99(\mathrm{~m}, 2 \mathrm{H}), 2.40(\mathrm{t}, J=6.6 \mathrm{~Hz}, 2 \mathrm{H}), 3.81(\mathrm{~s}, 3 \mathrm{H}), 5.98(\mathrm{~d}, J=10 \mathrm{~Hz}$, $1 \mathrm{H}), 6.99(\mathrm{ddd}, J=7.1,7.1,4.1 \mathrm{~Hz}, 1 \mathrm{H}) ;{ }^{13} \mathrm{C}-\mathrm{NMR}\left(125 \mathrm{MHz}, \mathrm{CDCl}_{3}\right): \delta 20.9,22.7,24.3,51.9,74.4,112.5$, $116.9,129.8,152.9$.

1-Cyano-1-methyl-3-phenylprope-2-yl methyl carbonate (4e) [81]: IR (neat): 2250, $1760 \mathrm{~cm}^{-1}$; ${ }^{1} \mathrm{H}-\mathrm{NMR}\left(500 \mathrm{MHz}, \mathrm{CDCl}_{3}\right): \delta 1.84(\mathrm{~s}, 3 \mathrm{H}), 3.72(\mathrm{~s}, 3 \mathrm{H}), 6.10(\mathrm{~d}, J=16.0 \mathrm{~Hz}, 1 \mathrm{H}), 6.95(\mathrm{~d}, J=16.0 \mathrm{~Hz}$, 1H), 7.29-7.30 (m, 3H), 7.43-7.44 (m, 2H); ${ }^{13} \mathrm{C}-\mathrm{NMR}\left(125 \mathrm{MHz}, \mathrm{CDCl}_{3}\right): \delta 14.2,21.1,55.4,60.4,73.8$, $117.3,124.0,128.8,130.5,134.4,152.9$.

1-Cyano-1-phenylethyl methyl carbonate (4f) [11]: IR (neat) 2250, $1752 \mathrm{~cm}^{-1} ;{ }^{1} \mathrm{H}-\mathrm{NMR}\left(500 \mathrm{MHz}, \mathrm{CDCl}_{3}\right)$ : $\delta 2.01(\mathrm{~s}, 3 \mathrm{H}), 3.77(\mathrm{~s}, 3 \mathrm{H}), 7.37-7.46(\mathrm{~m}, 3 \mathrm{H}), 7.53-7.55(\mathrm{~m}, 2 \mathrm{H}) ;{ }^{13} \mathrm{C}-\mathrm{NMR}\left(125 \mathrm{MHz}, \mathrm{CDCl}_{3}\right)$ : $\delta$ 29.9, $55.4,75.7,117.9,124.5,129.1,129.5,137.6,152.9$.

1-Cyano-1-phenylpropyl methyl carbonate (4g): pale yellow oil; IR (neat) 2249, $1758 \mathrm{~cm}^{-1}$; ${ }^{1} \mathrm{H}-\mathrm{NMR}\left(500 \mathrm{MHz}, \mathrm{CDCl}_{3}\right): \delta 1.03(\mathrm{t}, J=7.4 \mathrm{~Hz}, 3 \mathrm{H}), 2.05-2.12(\mathrm{~m}, 1 \mathrm{H}), 2.27-2.34(\mathrm{~m}, 1 \mathrm{H}), 3.74(\mathrm{~s}, 3 \mathrm{H})$, 
7.36-7.42 (m, 5H); ${ }^{13} \mathrm{C}-\mathrm{NMR}(125 \mathrm{MHz}, \mathrm{CDCl}): \delta 8.5,36.0,55.4,65.6,80.4,117.0,124.8,129.0$. HRMS (FAB): $m / z$ : calcd for $\mathrm{C}_{9} \mathrm{H}_{14} \mathrm{NO}_{3}$ : 220. 0968; found: $220.0979[\mathrm{M}+\mathrm{H}]^{+}$.

1-Cyano-1-(4-chlorophenyl)ethyl methyl carbonate (4h) [11]: IR (neat) 2250, $1760 \mathrm{~cm}^{-1} ;{ }^{1} \mathrm{H}-\mathrm{NMR}(500 \mathrm{MHz}$, $\left.\mathrm{CDCl}_{3}\right): \delta 1.99(\mathrm{~s}, 3 \mathrm{H}), 3.76(\mathrm{~s}, 3 \mathrm{H}), 7.38(\mathrm{~d}, J=8.7 \mathrm{~Hz}, 2 \mathrm{H}), 7.48(\mathrm{~d}, J=8.7 \mathrm{~Hz}, 2 \mathrm{H}) ;{ }^{13} \mathrm{C}-\mathrm{NMR}(125 \mathrm{MHz}$, $\left.\mathrm{CDCl}_{3}\right): \delta 29.7,55.5,66.0,75.1,117.5,126.1,129.3,135.6,136.1,152.8$.

1-Cyano-1-(4-nitrophenyl)ethyl methyl carbonate (4i) [11]: IR (neat) 2251, $1749 \mathrm{~cm}^{-1} ;{ }^{1} \mathrm{H}-\mathrm{NMR}(500 \mathrm{MHz}$, $\left.\mathrm{CDCl}_{3}\right): \delta 2.00(\mathrm{~s}, 3 \mathrm{H}), 3.76(\mathrm{~s}, 3 \mathrm{H}), 7.70(\mathrm{~d}, J=8.9 \mathrm{~Hz}, 2 \mathrm{H}), 8.06(\mathrm{~d}, J=8.9 \mathrm{~Hz}, 2 \mathrm{H}),{ }^{13} \mathrm{C}-\mathrm{NMR}(125 \mathrm{MHz}$, $\left.\mathrm{CDCl}_{3}\right): \delta 29.7,55.8,65.8,74.8,117.0,123.8,124.4,125.7,152.8$.

Cyano(phenyl)methyl methyl acetate (5a) [14]: IR (neat) 2250, $1742 \mathrm{~cm}^{-1} ;{ }^{1} \mathrm{H}-\mathrm{NMR}\left(500 \mathrm{MHz}, \mathrm{CDCl}_{3}\right)$ : $\delta 2.17(\mathrm{~s}, 3 \mathrm{H}), 6.39(\mathrm{~s}, 1 \mathrm{H}), 7.44(\mathrm{~m}, 3 \mathrm{H}), 7.51(\mathrm{~d}, J=11.1 \mathrm{~Hz}, 2 \mathrm{H}) ;{ }^{13} \mathrm{C}-\mathrm{NMR}\left(125 \mathrm{MHz}, \mathrm{CDCl}_{3}\right): \delta 20.4$, $62.8,116.1,127.9,129.2,130.4,131.7,168.9$.

Cyano(4-chlorophenyl)methyl methyl acetate (5b) [18]: IR (neat) 2245, $1756 \mathrm{~cm}^{-1},{ }^{1} \mathrm{H}-\mathrm{NMR}(500 \mathrm{MHz}$, $\left.\mathrm{CDCl}_{3}\right): \delta 2.15(\mathrm{~s}, 3 \mathrm{H}), 6.36(\mathrm{~s}, 1 \mathrm{H}), 7.41(\mathrm{~d}, J=8.7 \mathrm{~Hz}, 2 \mathrm{H}), 7.45(\mathrm{~d}, J=8.7 \mathrm{~Hz}, 2 \mathrm{H}) ;{ }^{13} \mathrm{C}-\mathrm{NMR}(125 \mathrm{MHz}$, $\left.\mathrm{CDCl}_{3}\right): \delta 20.4,62.1,115.7,129.3,129.5,130.2,136.6,168.8$.

Cyano(4-methylphenyl)methyl methyl acetate (5c) [18]: IR (neat) 2250, $1753 \mathrm{~cm}^{-1} ;{ }^{1} \mathrm{H}-\mathrm{NMR}(500 \mathrm{MHz}$, $\left.\mathrm{CDCl}_{3}\right): \delta 2.13(\mathrm{~s}, 3 \mathrm{H}), 2.37(\mathrm{~s}, 3 \mathrm{H}), 6.35(\mathrm{~s}, 1 \mathrm{H}), 7.23(\mathrm{~d}, J=8.0 \mathrm{~Hz}, 2 \mathrm{H}), 7.39(\mathrm{~d}, J=8.0 \mathrm{~Hz}, 2 \mathrm{H})$; ${ }^{13} \mathrm{C}-\mathrm{NMR}\left(125 \mathrm{MHz}, \mathrm{CDCl}_{3}\right)$ : $\delta 20.5,21.3,62.7,116.2,127.9,128.8,129.9,140.7,169.0$.

Cyano(4-methoxyphenyl)methyl methyl acetate (5d) [18]: IR (neat) 2249, $1747 \mathrm{~cm}^{-1} ;{ }^{1} \mathrm{H}-\mathrm{NMR}(500 \mathrm{MHz}$, $\left.\mathrm{CDCl}_{3}\right): \delta 2.12(\mathrm{~s}, 3 \mathrm{H}), 3.81(\mathrm{~s}, 3 \mathrm{H}), 6.34(\mathrm{~s}, 1 \mathrm{H}), 6.93(\mathrm{~d}, J=8.8 \mathrm{~Hz}, 2 \mathrm{H}), 7.43(\mathrm{~d}, J=8.8 \mathrm{~Hz}, 2 \mathrm{H})$; ${ }^{13} \mathrm{C}-\mathrm{NMR}\left(125 \mathrm{MHz}, \mathrm{CDCl}_{3}\right)$ : $\delta 20.5,55.4,62.6,114.5,116.3,123.8,129.6,161.1,169.0$.

Cyano(4-nitrophenyl)methyl methyl acetate (5e) [82]: IR (neat): 2251, $1760 \mathrm{~cm}^{-1} ;{ }^{1} \mathrm{H}-\mathrm{NMR}(500 \mathrm{MHz}$, $\left.\mathrm{CDCl}_{3}\right): \delta 2.20(\mathrm{~s}, 3 \mathrm{H}), 6.49(\mathrm{~s}, 1 \mathrm{H}), 7.71(\mathrm{~d}, J=8.7 \mathrm{~Hz}, 2 \mathrm{H}), 8.30(\mathrm{~d}, J=8.7 \mathrm{~Hz}, 2 \mathrm{H}) ;{ }^{13} \mathrm{C}-\mathrm{NMR}(125 \mathrm{MHz}$, $\left.\mathrm{CDCl}_{3}\right): \delta 20.3,61.6,115.1,124.4,128.7,138.1,149.0,168.6$.

Cyano(naphthalen-1-yl)methyl methyl acetate (5f) [18]: IR (neat) 2249, $1755 \mathrm{~cm}^{-1} ;{ }^{1} \mathrm{H}-\mathrm{NMR}(500 \mathrm{MHz}$, $\left.\mathrm{CDCl}_{3}\right): \delta 2.17(\mathrm{~s}, 3 \mathrm{H}), 7.02(\mathrm{~s}, 1 \mathrm{H}), 7.50(\mathrm{dd}, J=7.1,8.1 \mathrm{~Hz}, 1 \mathrm{H}), 7.54(\mathrm{t}, J=8.1 \mathrm{~Hz}, 1 \mathrm{H}), 7.60(\mathrm{t}, J=6.2 \mathrm{~Hz}$, $1 \mathrm{H}), 7.71(\mathrm{~d}, J=6.2 \mathrm{~Hz}, 1 \mathrm{H}), 7.86(\mathrm{~d}, J=7.1 \mathrm{~Hz}, 1 \mathrm{H}), 7.94(\mathrm{~d}, J=9.9 \mathrm{~Hz}, 1 \mathrm{H}), 7.98(\mathrm{~d}, J=8.6 \mathrm{~Hz}, 1 \mathrm{H})$; ${ }^{13} \mathrm{C}-\mathrm{NMR}\left(125 \mathrm{MHz}, \mathrm{CDCl}_{3}\right): \delta 20.4,61.3116 .1122 .5,125.0,126.6,126.9,127.6,129.1,130.0,131.5$, $133.9,169.0$.

Cyano(naphthalen-1-yl)methyl methyl acetate (5g) [82]: IR (neat) 2250, $1753 \mathrm{~cm}^{-1} ;{ }^{1} \mathrm{H}-\mathrm{NMR}(500 \mathrm{MHz}$, $\left.\mathrm{CDCl}_{3}\right): \delta 2.17(\mathrm{~s}, 3 \mathrm{H}), 6.57(\mathrm{~s}, 1 \mathrm{H}), 7.53-7.57(\mathrm{~m}, 3 \mathrm{H}), 7.87(\mathrm{t}, J=9.3 \mathrm{~Hz}, 2 \mathrm{H}), 7.91(\mathrm{~d}, J=8.6 \mathrm{~Hz}, 1 \mathrm{H})$, $8.00(\mathrm{~d}, J=1.3 \mathrm{~Hz}, 1 \mathrm{H}) ;{ }^{13} \mathrm{C}-\mathrm{NMR}\left(125 \mathrm{MHz}, \mathrm{CDCl}_{3}\right): \delta 20.6,63.1,116.2,124.3,127.1,127.6,127.1,127.6$, $127.9,128.1,128.4,129.5,132.9,133.9,169.0$.

Cyano-3-phenylpropen-2-yl methyl acetate (5h) [32]: IR (neat) 2251, $1751 \mathrm{~cm}^{-1} ;{ }^{1} \mathrm{H}-\mathrm{NMR}(500 \mathrm{MHz}$, $\left.\mathrm{CDCl}_{3}\right): \delta 2.15(\mathrm{~s}, 3 \mathrm{H}), 6.00(\mathrm{~d}, J=6.7 \mathrm{~Hz}, 1 \mathrm{H}), 6.18(\mathrm{dd}, J=6.7,15.8 \mathrm{~Hz}, 1 \mathrm{H}), 6.95(\mathrm{~d}, J=15.8 \mathrm{~Hz}, 1 \mathrm{H})$, 7.31-7.37 (m, 3H), $7.41(\mathrm{~d}, J=7.9 \mathrm{~Hz}, 2 \mathrm{H}) ;{ }^{13} \mathrm{C}-\mathrm{NMR}\left(125 \mathrm{MHz}, \mathrm{CDCl}_{3}\right): \delta 20.3,61.4,115.4,118.2,127.0$, $128.7,129.3,134.3,137.7,168.8$.

Cyano-3-phenylpropeyl methyl acetate (5i) [18]: IR (neat) 2248, $1755 \mathrm{~cm}^{-1} ;{ }^{1} \mathrm{H}-\mathrm{NMR}\left(500 \mathrm{MHz}, \mathrm{CDCl}_{3}\right.$ ): $\delta 2.11(\mathrm{~s}, 3 \mathrm{H}), 2.22(\mathrm{dd}, J=7.6,15.0 \mathrm{~Hz}, 2 \mathrm{H}), 2.82(\mathrm{t}, J=7.6 \mathrm{~Hz}, 2 \mathrm{H}), 5.25(\mathrm{t}, J=6.8 \mathrm{~Hz}, 1 \mathrm{H})$, $7.17(\mathrm{~d}, J=7.2 \mathrm{~Hz}, 2 \mathrm{H}), 7.23(\mathrm{~d}, J=8.1 \mathrm{~Hz}, 1 \mathrm{H}), 7.30(\mathrm{t}, J=7.2 \mathrm{~Hz}, 2 \mathrm{H}) ;{ }^{13} \mathrm{C}-\mathrm{NMR}\left(125 \mathrm{MHz}, \mathrm{CDCl}_{3}\right)$ : $\delta 20.3,30.7,33.7,60.5,116.7,126.7,128.3,128.7,139.0,169.1$. 
Cyano(cyclohexyl)methyl methyl acetate (5j) [18]: IR (neat) 2248, $1755 \mathrm{~cm}^{-1} ;{ }^{1} \mathrm{H}-\mathrm{NMR}\left(500 \mathrm{MHz}, \mathrm{CDCl}_{3}\right)$ : $\delta$ 1.07-1.21 (m, 4H), 1.21-1.30 (m, 2H), 1.66-1.69 (m, 1H), 1.73-1.80 (m, 3H), 1.84-1.92 (m, 1H), $2.13(\mathrm{~s}, 3 \mathrm{H}), 5.15(\mathrm{~d}, J=6.6 \mathrm{~Hz}, 1 \mathrm{H}) ;{ }^{13} \mathrm{C}-\mathrm{NMR}\left(125 \mathrm{MHz}, \mathrm{CDCl}_{3}\right): \delta$ 20.3, 25.3, 25.7, 28.1, 40.0, 65.6, $116.2,169.2$.

Cyano(1-octyl)methyl methyl acetate (5k) [83]: IR (neat) 2218, $1756 \mathrm{~cm}^{-1},{ }^{1} \mathrm{H}-\mathrm{NMR}\left(500 \mathrm{MHz}, \mathrm{CDCl}_{3}\right)$ : $\delta 0.83(\mathrm{t}, J=7.0 \mathrm{~Hz}, 3 \mathrm{H}), 1.15-1.33(\mathrm{~m}, 14 \mathrm{H}), 1.57$ (quin, $J=6.8 \mathrm{~Hz}, 2 \mathrm{H}), 1.99(\mathrm{~s}, 3 \mathrm{H}), 4.00(\mathrm{t}, J=6.8 \mathrm{~Hz}$, 2H); ${ }^{13} \mathrm{C}-\mathrm{NMR}\left(125 \mathrm{MHz}, \mathrm{CDCl}_{3}\right): \delta$ 14.0, 20.9, 22.6, 25.8, 28.5, 29.2, 29.4, 31.8, 64.6, 117.6, 171.1.

Cyano(furan-2-yl)methyl methyl acetate (5l) [82]: IR (neat) 2248, $1753 \mathrm{~cm}^{-1} ;{ }^{1} \mathrm{H}-\mathrm{NMR}\left(500 \mathrm{MHz}, \mathrm{CDCl}_{3}\right)$ : $\delta 2.14(\mathrm{~s}, 3 \mathrm{H}), 6.42(\mathrm{dd}, J=1.9,3.4 \mathrm{~Hz}, 1 \mathrm{H}), 6.45(\mathrm{~s}, 1 \mathrm{H}), 6.66(\mathrm{~d}, J=3.4 \mathrm{~Hz}, 1 \mathrm{H}), 7.49(\mathrm{dd}, J=0.8,1.9 \mathrm{~Hz}$, $1 \mathrm{H}) ;{ }^{13} \mathrm{C}-\mathrm{NMR}\left(125 \mathrm{MHz}, \mathrm{CDCl}_{3}\right): \delta 20.3,55.7,111.0,112.6,114.1,144.1,145.0,168.7$.

\section{Conclusions}

In conclusion, we have demonstrated the TBD-catalyzed cyanation reaction of carbonyl compounds using methyl cyanoformate or acetyl cyanide as cyanide sources. A broad range of aldehydes and ketones reacted to produce $O$-protected cyanohydrins in the presence of 5-10 mol \% of TBD. Furthermore, a polymer-supported catalyst, PS-TBD, also acted as a good catalyst for this reaction. PS-TBD was easily recovered and reused with minimal loss of activity. These reactions provide a simple and convenient method for synthesis of highly functionalized $\alpha$-hydroxyl-carbonyl compounds and $\beta$-amino alcohols.

Supplementary Materials: Supplementary materials can be accessed at: http:/ /www.mdpi.com/1420-3049/21/ 8/1030/s1.

Author Contributions: S.M. conceived the ideas, analyzed the data, and wrote the paper; J.K and M.Y. performed the experiments and analyzed the data; and all authors read and approved the final manuscript.

Conflicts of Interest: The authors declare no conflict of interest.

\section{References}

1. North, M. Catalytic, asymmetric cyanohydrin formation. Synlett 1993, 1993, 807-820. [CrossRef]

2. Gregory, J.H. Cyanohydrins in nature and the laboratory: Biology, preparations, and synthetic applications. Chem. Rev. 1999, 99, 3649-3682. [CrossRef] [PubMed]

3. North, M. Synthesis and applications of non-racemic cyanohydrins. Tetrahedron Asymmetry 2003, 14, 147-176. [CrossRef]

4. Brunel, J.M.; Holmes, I.P. Chemically catalyzed asymmetric cyanohydrin syntheses. Angew. Chem. Int. Ed. 2004, 43, 2752-2778. [CrossRef] [PubMed]

5. Chen, F.X.; Feng, X. Synthesis of racemic tertiary cyanohydrins. Synett 2005, 2005, 892-899. [CrossRef]

6. Khan, N.H.; Kureshy, R.I.; Abdi, S.H.R.; Agrawal, S.; Jasra, R. Metal catalyzed asymmetric cyanation reactions. Coord. Chem. Rev. 2008, 252, 593-623. [CrossRef]

7. Evans, D.A.; Truesdale, L.K. Carbonyl insertion reactions of silicon pseudohalides: Catalysis. Tetrahedron Lett. 1973, 14, 4929-4932. [CrossRef]

8. Evans, D.A.; Carroll, G.L.; Truesdale, L.K. Synthetic applications of trimethylsilyl cyanide. An efficient synthesis of aminomethyl alcohols. J. Org. Chem. 1974, 39, 914-917. [CrossRef]

9. Scholl, M.; Lim, C.K.; Fu, G.C. Convenient and efficient conversion of aldehydes to acylated cyanohydrins using tributyltin cyanide as a catalyst. J. Org. Chem. 1995, 60, 6229-6231. [CrossRef]

10. Okimoto, M.; Chiba, T. A Convenient and Improved Method for the Preparation of Cyanohydrin Esters from Acyl Cyanides and Aldehydes. Synthesis 1996, 1996, 1188-1190. [CrossRef]

11. Baeza, A.; Najera, C.; Retamosa, M.G.; Sansano, J.M. Solvent-free synthesis of cyanohydrin derivatives catalysed by triethylamine. Synthesis 2005, 2005, 2787-2797.

12. Poirier, D.; Berthiaume, D.; Boivi, R.P. Unexpected formation of O-methoxycarbonyl cyanohydrin showing potential as a protective group of ketones. Synlett 1999, 1999e, 1423-1425. [CrossRef] 
13. Deardorff, D.R.; Taniguchi, C.M.; Tafti, S.A.; Kim, H.Y.; Choi, S.Y.; Downey, K.J.; Nguyen, T.V. A two-Step procedure for the conversion of $\alpha, \beta$-Unsaturated aldehydes into $\gamma$-Azido- $\alpha, \beta$-unsaturated nitriles. J. Org. Chem. 2001, 66, 7191-7194. [CrossRef] [PubMed]

14. Hoffmann, H.M.R.; Ismail, Z.M.; Hollweg, R.; Zein, A.R. DABCO-mediated synthesis and biological activity of cyanohydrin esters. Bull. Chem. Soc. Jpn. 1990, 63, 1807-1810. [CrossRef]

15. Iwanami, K.; Hinakubo, Y.; Oriyama, T. Catalyst-free DMSO-promoted synthesis of cyanohydrin carbonates from aldehydes. Tetrahedron Lett. 2005, 46, 5881-5883. [CrossRef]

16. Matsukawa, S.; Sekine, I.; Iitsuka, A. Tris(2,4,6-trimethoxyphenyl) phosphine (TTMPP): Efficient Catalysts for the Cyanosilylation and Cyanocarbonation of Aldehydes and Ketones. Molecules 2009, 14, 3353-3359. [CrossRef] [PubMed]

17. Aoki, S.; Kotani, S.; Sugiura, M.; Nakajima, M. DMAP-catalyzed cyanation of aldehydes and ketones with ethyl cyanoformate. Tetrahedron Lett. 2010, 51, 3547-3549. [CrossRef]

18. Zhang, J.; Du, G.; Xu, Y.; He, L.; Dai, B. N-heterocyclic carbene catalyzed cyanation reaction of carbonyl compounds with ethyl cyanoformate and acetyl cyanide. Tetrahedron Lett. 2011, 52, 7153-7156. [CrossRef]

19. Tian, S.K.; Deng, L. A Highly enantioselective chiral Lewis base-catalyzed asymmetric cyanation of ketones. J. Am. Chem. Soc. 2001, 123, 6195-6196. [CrossRef] [PubMed]

20. Tian, S.K.; Deng, L. Enantioselective cyanocarbonation of ketones with chiral base. Tetrahedron 2006, 62, 11320-11330. [CrossRef]

21. Chinchilla, R.; Nájera, C.; Ortega, F.J. Enantioselective cyanoformylation of aldehydes using a recyclable dimeric cinchonidine ammonium salt as an organocatalyst. Tetrahedron Asymmetry 2008, 19, 265-268. [CrossRef]

22. Li, F.; Widyan, K.; Wingstrand, E.; Moberg, C. Chiral Lewis base catalyzed enantioselective acetylcyanation of $\alpha$-oxo esters. Eur. J. Org. Chem. 2009, 2009, 3917-3922. [CrossRef]

23. Wang, J.; Wang, W.; Li, W.; Hu, X.; Shen, K.; Tan, C.; Liu, X.; Feng, X. Asymmetric Cyanation of Aldehydes, Ketones, Aldimines, and Ketimines Catalyzed by a Versatile Catalyst Generated from Cinchona Alkaloid, Achiral Substituted 2,2'-Biphenol and Tetraisopropyl Titanate. Chem. Eur. J. 2009, 15, 11642-11659. [CrossRef] [PubMed]

24. Ogura, Y.; Akakura, M.; Sakakura, A.; Ishihara, K. Enantioselective cyanoethoxycarbonylation of Isatins promoted by a Lewis base-Brønsted acid cooperative catalyst. Angew. Chem. Int. Ed. 2013, 52, 8299-8303. [CrossRef] [PubMed]

25. Ishihara, K.; Ogura, Y. Enantioselective Cyano-Alkoxycarbonylation of $\alpha$-Oxoesters Promoted by Brønsted Acid-Lewis Base Cooperative Catalysts. Org. Lett. 2015, 17, 6070-6073. [CrossRef] [PubMed]

26. Tian, J.; Yamagiwa, N.; Matsunaga, S.; Shibasaki, M. An asymmetric cyanation reaction and sequential asymmetric cyanation-nitroaldol reaction using a $\left[\mathrm{YLi}_{3}\{\right.$ tris(binaphthoxide)\}] single catalyst component: catalyst tuning with achiral additives. Angew. Chem. Int. Ed. 2002, 41, 3636-3638. [CrossRef]

27. Tian, J.; Yamagiwa, N.; Matsunaga, S.; Shibasaki, M. Efficient two-step conversion of $\alpha, \beta$-unsaturated aldehydes to optically active $\gamma$-oxy- $\alpha, \beta$-unsaturated nitriles and its application to the total synthesis of (+)-Patulolide C. Org. Lett. 2003, 5, 3021-3024. [CrossRef] [PubMed]

28. Yamagiwa, N.; Tian, J.; Matsunaga, S.; Shibasaki, M. Catalytic asymmetric cyano-ethoxycarbonylation reaction of aldehydes using a $\mathrm{YLi}_{3}$ Tris (binaphthoxide) (YLB) complex: Mechanism and roles of achiral additives. J. Am. Chem. Soc. 2005, 127, 3413-3422. [CrossRef] [PubMed]

29. Casas, J.; Baeza, A.; Sansano, J.M.; Nájera, C.; Saá, J.M. Enantioselective cyanoformylation of aldehydes mediated by BINOLAM-AlCl as a monometallic bifunctional catalys. Tetrahedron Asymmetry 2003, 14, 197-200. [CrossRef]

30. Baeza, A.; Casas, J.; Nájera, C.; Sansano, J.M.; Saá, J.M. Enantioselective synthesis of O-methoxycarbonyl cyanohydrins: Chiral building blocks generated by bifunctional catalysis with BINOLAM-AlCl. Eur. J. Org. Chem. 2006, 2006e, 1949-1958. [CrossRef]

31. Belokon', Y.N.; Blacker, A.J.; Clutterbuck, L.A.; North, M. Catalytic, asymmetric synthesis of cyanohydrin ethyl carbonates. Org. Lett. 2003, 5, 4505-4507. [CrossRef] [PubMed]

32. Lundgren, S.; Wingstrand, E.; Penhoat, M.; Moberg, C. Dual Lewis Acid-Lewis Base activation in enantioselective cyanation of aldehydes using acetyl cyanide and cyanoformate as cyanide sources. J. Am. Chem. Soc. 2005, 127, 11592-11593. [CrossRef] [PubMed] 
33. Li, Q.; Chang, L.; Liu, X.; Feng, X. Catalytic asymmetric cyano-ethoxycarbonylation reaction of aldehydes using a novel C2-symmetric chiral $N, N^{\prime}$-dioxide Titanium complex. Synlett 2006, 1675-1678. [CrossRef]

34. Gou, S.; Chen, X.; Xiong, Y.; Feng, X. Highly Enantioselective cyanation of aldehydes catalyzed by a multicomponent Titanium complex. J. Org. Chem. 2006, 71, 5732-5736. [CrossRef] [PubMed]

35. Chen, S.K.; Peng, D.; Zhou, H.; Wang, L.W.; Chen, F.X.; Feng, X.M. Highly enantioselective cyanoformylation of aldehydes catalyzed by a mononuclear Salen-Ti(OiPr) $)_{4}$ complex produced in situ. Eur. J. Org. Chem. 2007, 2007, 639-644. [CrossRef]

36. Gou, S.; Liu, X.; Zhou, X.; Feng, X. Asymmetric cyano-ethoxycarbonylation of aldehydes catalyzed by self-assembled titanium catalyst. Tetrahedron 2007, 63, 7935-7941. [CrossRef]

37. Lundgren, S.; Wingstrand, E.; Moberg, C. Lewis Acid-Lewis Base-Catalysed Enantioselective Addition of a-Ketonitriles to Aldehydes. Adv. Synth. Catal. 2007, 349, 364-372. [CrossRef]

38. Belokon', Y.N.; Clegg, W.; Harrington, R.W.; Ishibashi, E.; Nomura, H.; North, M. Enantioselective and diastereoselective syntheses of cyanohydrin carbonates. Tetrahedron 2007, 63, 9724-9740. [CrossRef]

39. Wingstrand, E.; Laurell, A.; Fransson, L.; Hult, K.; Moberg, C. Minor Enantiomer Recycling: Metal Catalyst, Organocatalyst and Biocatalyst Working in Concert. Chem. Eur. J. 2009, 14, 12107-12113. [CrossRef] [PubMed]

40. Ji, N.; Yao, L.; He, W.; Li, Y. Bifunctional Schiff base/Ti (IV) catalysts for enantioselective cyanoformylation of aldehydes with ethyl cyanoformate. Appl. Organomet. Chem. 2013, 27, 209-213. [CrossRef]

41. Khan, N.U.H.; Agrawal, S.; Kureshy, R.I.; Abdi, S.H.R.; Pathak, K.; Bajaj, H.C. Enantioselective cyanoformylation of aldehydes catalyzed with solid base mediated chiral V (V) salen complexes. Chirality 2010, 22, 153-158. [CrossRef] [PubMed]

42. Schroeder, G.; Łeska, B.; Jarczewski, A.; Nowak-Wydra, B.; Brzezinski, B. FTIR, NMR and kinetic studies of proton transfer reactions from nitro-substituted diarylmethanes to $\mathrm{N}$-bases with guanidine character. J. Mol. Struct. 1995, 344, 77-88. [CrossRef]

43. Coles, M.P. Bicyclic-guanidines, -guanidinates and -guanidinium salts: Wide ranging applications from a simple family of molecules. Chem. Commun. 2009, 2009, 3659-3676. [CrossRef] [PubMed]

44. Simoni, D.; Rondanin, R.; Morini, M.; Baruchello, R.; Invidata, F.P. 1,5,7-Triazabicyclo [4.4.0] dec-1-ene (TBD), 7-methyl-TBD (MTBD) and the polymer-supported TBD (P-TBD): Three efficient catalysts for the nitroaldol (Henry) reaction and for the addition of dialkyl phosphites to unsaturated systems. Tetrahedron Lett. 2000, 41, 1607-1610. [CrossRef]

45. Simoni, D.; Rossi, M.; Rondanin, R.; Mazzali, A.; Baruchello, R.; Malagutti, C.; Roberti, M.; Invidata, F.P. Strong bicyclic guanidine base-promoted Wittig and Horner-Wadsworth-Emmons reactions. Org. Lett. 2000, 2, 3765-3768. [CrossRef] [PubMed]

46. Ye, W.; Xu, J.; Tan, C.T.; Tan, C.H. 1,5,7-Triazabicyclo [4.4.0]dec-5-ene (TBD) catalyzed Michael reactions. Tetrahedron Lett. 2005, 46, 6875-6878. [CrossRef]

47. Deredas, D.; Huben, K.; Maniukiewicz, W.; Krawczyk, H. Highly syn-diastereoselective Michael addition of enolizable ketones to 3-(diethoxyphosphoryl)coumarin. Tetrahedron 2014, 70, 8925-8929. [CrossRef]

48. Ghobril, C.; Sabot, C.; Mioskowski, C.; Baati, R. TBD-Catalyzed Direct 5- and 6-enolexo Aldolization of Ketoaldehydes. Eur. J. Org. Chem. 2008, 2008, 4104-4108. [CrossRef]

49. Lanari, D.; Rosati, O.; Curini, M. A solvent-free protocol for the synthesis of 3-formyl-2H-chromenes via domino oxa Michael/aldol reaction. Tetrahedron Lett. 2014, 55, 1752-1755. [CrossRef]

50. Pratt, R.C.; Lohmeijer, B.G.G.; Long, D.A.; Waymouth, R.A.; Hedric, J.A. Triazabicyclodecene: A simple bifunctional organocatalyst for acyl transfer and ring-opening polymerization of cyclic esters. J. Am. Chem. Soc. 2006, 129, 4556-4557. [CrossRef] [PubMed]

51. Simón, L.; Goodman, J.M. The Mechanism of TBD-catalyzed ring-opening polymerization of cyclic esters. J. Org. Chem. 2007, 72, 9656-9662. [CrossRef] [PubMed]

52. Sabot, C.; Kumar, K.A.; Meunier, S.; Mioskowski, C. A convenient aminolysis of esters catalyzed by 1,5,7-triazabicyclo[4.4.0]dec-5-ene (TBD) under solvent-free conditions. Tetrahedron Lett. 2007, 48, 3863-3866. [CrossRef]

53. Sabot, C.; Kumar, K.A.; Antheaume, C.; Mioskowski, C. Triazabicyclodecene: An Effective Isotope Exchange Catalyst in $\mathrm{CDCl}_{3}$. J. Org. Chem. 2007, 72, 5001-5004. [CrossRef] [PubMed]

54. Jiang, Z.; Zhang, Y.; Ye, W.; Tan, C.H. P-C Bond formation via direct and three-component conjugate addition catalyzed by 1,5,7-triazabicyclo [4.4.0]dec-5-ene (TBD). Tetrahedron Lett. 2007, 48, 51-54. [CrossRef] 
55. Mahe, O.; Frath, D.; Dez, I.; Marsais, F.; Levacher, V.; Briere, J.F. TBD-organocatalysed synthesis of pyrazolines. Org. Biomol. Chem. 2009, 7, 3648-3651. [CrossRef] [PubMed]

56. Saliu, F.; Rindone, B. Organocatalyzed synthesis of ureas from amines and ethylene carbonate. Tetrahedron Lett. 2010, 51, 6301-6304. [CrossRef]

57. Poladura, B.; Martínez-Castañeda, Á.; Rodríguez-Solla, H.; Concellón, C.; del Amo, V. TBD-catalyzed $\alpha$-sulfenylation of cyclic ketones: Desymmetrization of 4-substituted cyclohexanone. Tetrahedron 2012, 68, 6438-6446. [CrossRef]

58. Kiesewetter, M.K.; Scholten, M.; Kirn, N.; Weber, R.L.; Hedrick, J.L.; Waymouth, R.M. Cyclic guanidine organic catalysts: What is magic about triazabicyclodecene? J. Org. Chem. 2009, 74, 9490-9496. [CrossRef] [PubMed]

59. Hammar, P.; Ghobril, C.; Antheaume, C.; Wagner, A.; Baati, R.; Himo, F. Theoretical Mechanistic Study of the TBD-Catalyzed Intramolecular aldol reaction of ketoaldehydes. J. Org. Chem. 2010, 75, 4728-4736. [CrossRef] [PubMed]

60. Fu, X.; Tan, C.H. Mechanistic considerations of guanidine-catalyzed reactions. Chem. Commun. 2011, 47, 8210-8222. [CrossRef] [PubMed]

61. Matsukawa, S.; Harada, T.; Takahashi, H. TBD-catalyzed ring opening of aziridines with silylated nucleophiles. Synth. Commun. 2013, 43, 406-414. [CrossRef]

62. Matsukawa, S.; Takahashi, H.; Takahashi, S. TBD-catalyzed trifluoromethylation of carbonyl compounds with (trifluoromethyl)trimethylsilane. Synth Commun. 2013, 43, 1523-1529. [CrossRef]

63. Matsukawa, S.; Mouri, Y. A mild and regioselective ring-opening of aziridines with acid anhydride using TBD or PS-TBD as a catalyst. Molecules 2015, 20, 18482-18495. [CrossRef] [PubMed]

64. Tomoi, M.; Kato, Y.; Kakiuchi, H. Polymer-supported bases, 2. Polystyrene-supported 1,8-diazabicyclo[5.4.0] undec-7-ene as reagent in organic syntheses. Makromol. Chem. 1984, 185, 2117-2124. [CrossRef]

65. Iijima, K.; Fukuda, W.; Tomoi, M. Polymer-supported bases. XI. Esterification and alkylation in the presence of polymer-supported bicyclic amidine or guanidine moieties. J. Macromol. Sci. A Pure Appl. Chem. 1992, 29, 249-261. [CrossRef]

66. Xu, W.; Mohan, R.; Morrissey, M.M. Polymer supported bases in combinatorial chemistry: Synthesis of aryl ethers from phenols and alkyl halides and aryl halides. Tetrahedron. Lett. 1997, 38, 7337-7340. [CrossRef]

67. Boisnard, S.; Chastanet, J.; Zhu, J. A high throughput synthesis of aryl triflate and aryl nonaflate promoted by a polymer supported base (PTBD). Tetrahedron Lett. 1999, 40, 7469-7472. [CrossRef]

68. McNamara, C.A.; Dixon, M.J.; Bradley, M. Recoverable catalysts and reagents using recyclable polystyrene-based supports. Chem. Rev. 2002, 102, 3275-3300. [CrossRef] [PubMed]

69. Benaglia, M.; Puglisi, A.; Cozzi, F. Polymer-supported organic catalysts. Chem. Rev. 2003, 103, 3401-3429. [CrossRef] [PubMed]

70. Cozzi, F. Immobilization of organic catalysts: When, why, and how. Adv. Synth. Catal. 2006, 348, 1367-1390. [CrossRef]

71. Ikegami, S.; Hamamoto, H. Novel recycling system for organic synthesis via designer polymer-gel catalysts. Chem. Rev. 2009, 109, 583-593. [CrossRef] [PubMed]

72. Lu, J.; Toy, P.H. Organic polymer supports for synthesis and for reagent and catalyst immobilization. Chem. Rev. 2009, 109, 815-838. [CrossRef] [PubMed]

73. Kristensen, T.E.; Hansen, T. Polymer-supported chiral organocatalysts: Synthetic strategies for the road towards affordable polymeric immobilization. Eur. J. Org. Chem. 2010, 17, 3179-3204. [CrossRef]

74. Fringuelli, F.; Pizzo, F.; Vittoriani, C.; Vaccaro, L. Polystyryl-supported TBD as an efficient and reusable catalyst under solvent-free conditions. Chem. Commun. 2004, 2004, 2756-2757. [CrossRef] [PubMed]

75. Fringuelli, F.; Pizzo, F.; Vittoriani, C.; Vaccaro, L. Polystyrene-supported 1,5,7-triazabicyclo[4.4.0]dec-5-ene as an efficient and reusable catalyst for the thiolysis of 1,2-epoxides under solvent-free conditions. Eur. J. Org. Chem. 2006, 2006, 1231-1236. [CrossRef]

76. Lanari, D.; Balini, R.; Palmieri, A.; Pizzo, F.; Vaccaro, L. Diastereoselective three-step route to $o$-(6-nitrocyclohex-3-en-1-yl) phenol and tetrahydro-6H-benzo[c]chromen-6-ol derivatives from salicylaldehydes. Eur. J. Org. Chem. 2011, 2011, 2874-2884. [CrossRef]

77. Matsukawa, S.; Fujikawa, S. Polystyrene-supported TBD as an efficient and reusable organocatalyst for cyanosilylation of aldehydes, ketones, and imines. Tetrahedron Lett. 2012, 53, 1075-1077. [CrossRef] 
78. Matsukawa, S.; Harada, T.; Yasuda, S. Polystyrene-supported TBD catalyzed ring-opening of $N$-tosylaziridines with silylated nucleophiles. Org. Biomol. Chem. 2012, 10, 4886-4890. [CrossRef] [PubMed]

79. Matsukawa, S.; Tsukamoto, K.; Harada, T.; Yasuda, S. An efficient method for opening $N$-tosyl aziridines with silylated nucleophiles using polystyrene-supported TBD as a reusable organocatalyst. Synthesis 2013, 45, 2959-2965. [CrossRef]

80. Berthiaume, D.; Poirier, D. O-Methoxycarbonyl Cyanohydrin as a New Protective Group for Carbonyls. Tetrahedron 2000, 56, 5995-6003. [CrossRef]

81. Hünig, S.; Reichelt, H. Trimethylsilylcyanid als umpolungsreagens, XI. Über den ambidenten charakter substituierter allyl-anionen. Chem. Ber. 1986, 119, 1772-1800. [CrossRef]

82. Kadam, S.T.; Kim, S.S. One-pot three components synthesis of O-acetylcyanohydrins with TMSCN, acetic anhydride and carbonyl compounds under solvent-free condition. Tetrahedron 2009, 65, 6330-6334. [CrossRef]

83. Quin, Y.C.; Liu, L.; Pu, L. On-step synthesis of a bifunctional BINOL ligand for the highly enantioselective cyanation of aliphatic aldehydes. Org. Lett. 2005, 7, 2381-2383. [CrossRef] [PubMed]

Sample Availability: Samples of the compounds $\mathbf{2 a - 2 j}, \mathbf{4 a - 4 i}, \mathbf{5 a}-\mathbf{5 l}$ are available from the authors.

(C) 2016 by the authors; licensee MDPI, Basel, Switzerland. This article is an open access article distributed under the terms and conditions of the Creative Commons Attribution (CC-BY) license (http:/ / creativecommons.org/licenses/by/4.0/). 